\title{
LaTeoría de la Educación: Una Búsqueda sin Término en la Construcción del Conocimiento de la Educación
}

\author{
Rafael Sáez Alonso \\ Universidad Complutense de Madrid
}

\section{ABSTRACT}

Knowledge of educational issues is construed using very different sources which, in turn, produce different forms of knowledge. Theory of education constitutes a fundamental source in the building of knowledge of educational matters. This essay makes the case that theory of education plays a special role in educational research and it shows how educational theory plays a specific role by enabling us to approach educational problems with our own criteria. Further, educational theory has both epistemological and ontological bases, the former referring to the way of knowing and the latter to the reality it studies. Thus, it sustains education making it susceptible to be studied with functional autonomy, using and developing the reality that is education as an object and a goal. Here, the author argues that educational theory faces the challenge of remaining open to research to favour its development as a scientific construction and academic discipline, in the form of either research about education as part of reality, or research into the knowledge of education.

Key words: education, educational Theory, globalisation, knowledge of education, research paradigms.

\section{RESUMEN}

El conocimiento en relación a la educación se construye con diferentes fuentes epistemológicas que, a su vez, producen diferentes formas de conocimiento. La teoría de la educación tiene un papel específico en la investigación educativa y posee un dominio para abordar los problemas de educación con criterios propios, pues está fundamentada epistemológicamente, por la forma de conocer y ontológicamente, por el ámbito de la realidad que estudia. Por eso, sustenta a la educación y la hace susceptible de estudio con sentido de autonomía funcional, utilizando y desarrollando el ámbito de realidad que es la educación como objeto y como meta de su quehacer. El autor muestra que la teoría de la educación tiene el reto de permanecer abierta a la investigación para favorecer su desarrollo como construcción científica y como disciplina académica bien sea como investigación acerca de la educación como ámbito de realidad, bien sea como investigación del conocimiento de la educación.

Descriptores: educación, teoría de la educación, globalización, conocimiento de la educación, paradigmas de investigación. 


\section{RÉSUMÉ}

L'objectif de ce travail consiste à mettre l'accent sur le fait que la connaissance de l'éducation provient de sources de connaissance très diverses qui, à leur tour, produisent différentes formes de cette dernière. Une source primaire qui génère la connaissance éducative est la théorie de l'éducation et fait partie de la connaissance de l'éducation. Il est montré dans cet article la manière dont la théorie de l'éducation joue un rôle spécifique dans la recherche éducative et possède un domaine pour aborder les problèmes d'éducation sur la base de critères propres, car elle prend appui, du point de vue épistémologique, sur la manière de connaître et du point de vue ontologique, sur le champ de la réalité qu'elle étudie. C'est pourquoi, elle soutient l'éducation et fait qu'elle soit susceptible d'étude avec un sens d'autonomie fonctionnelle, en utilisant et en développant le champ de réalité qu'est l'éducation comme objet et comme objectif de son propos. L' auteur justifie que la théorie de l'éducation présente le défi d'être ouverte à la recherche pour favoriser son développement comme construction scientifique et comme discipline académique, soit comme recherche sur l'éducation comme champ de réalité, soit comme recherche de la connaissance de l'éducation.

Mots-clés: éducation, théorie de l'éducation, globalisation, connaissance de l'éducation, paradigmes de recherche.

\section{Introducción}

【 A TEORÍA DE LA EDUCACIÓN hace esfuerzos permanentes para cuestionarse y reflexionar sobre sí misma. En la Pedagogía, las preguntas, ¿qué es la Educación?, ¿qué es la Teoría de la Educación?, juegan un papel inherente y esencial a la misma investigación educativa.

A lo largo de los últimos años ha aparecido una abundante reflexión sobre esta temática. Y esto hace referencia, sin duda, a que el pensamiento sobre los presupuestos de la teoría de la educación es recursivo en todas sus direcciones y sentidos de la investigación pedagógica. Este pensamiento recursivo importa más, si cabe, que en otras ramas del saber, como la Física o las Matemáticas.

Como decíamos en otro trabajo, "la educación es, por naturaleza y desde el principio, también reflexión no sólo sobre el quehacer educativo, sino también sobre la educación, o mejor, sobre la teoría de la educación” (Sáez Alonso, 1994, p. 231). Marcar los límites y definir la teoría de la educación es una empresa que necesariamente lleva a analizar la evolución del conocimiento de la Educación. Y, como dice el profesor Touriñán, esto se afirma desde la firme convicción compartida por muchos de que la teoría tiene un papel específico en la investigación educativa, bien sea como investigación acerca de la educación como ámbito de realidad, bien sea como investigación del conocimiento de la educación (Touriñán, 2007, p.2).

Así mismo, el mundo global en que vivimos y su influencia en la vida de las personas obliga a ser recursivos y preguntarnos, una vez más, ¿qué es la teoría de la educación? La globalización, término con una complejidad semántica muy abundante adquiere una presencia total en la <aldea global> en que se ha convertido la sociedad actual:

"Si creemos que ésta (la educación) debe servir a un proyecto de ser humano y de sociedad, tendremos que aprovechar las posibilidades y afrontar los riesgos de la globalización formando sujetos que la puedan reorientar. Educar para la vida es educar para un mundo en el que nada nos es ajeno. La educación se ve 
necesariamente obligada a replantear sus metas y a revisar sus contenidos y métodos" (Gimeno Sacristán, 2001, p. 121).

La visión de un mundo homogéneo y uniforme está desapareciendo. La globalización afecta en su misma raíz a los individuos y a la sociedad en todos los planos: político, económico, cultural y socio-educativo. Hay una afinidad entre las distintas globalizaciones, aunque no sean reducibles unas a otras, ni explicables unas por otras, pero sí explicables todas ellas por la sociedad de la información, la mundialización y la civilización que favorecen el carácter abierto de la sociedad del conocimiento que define a la sociedad actual científico-técnica.

El sesgo propio de la globalización, la civilización científico-técnica, la sociedad de la información y la mundialización de los fenómenos, junto con el sentido democrático de las sociedades abiertas que se manifiesta en la participación, la autonomía y el reconocimiento y respeto al otro, configuran el nuevo marco de pensamiento que justifica el planteamiento de una reflexión sobre la educación en nuestro mundo, desde la teoría de la educación, que dicho sea de paso y a modo de presupuesto, no cambia conceptualmente como disciplina que indaga el conocimiento de la educación por el hecho de estar encuadrada en un mundo globalizante, aunque puede modificar su contenido al abordar la educación como ámbito de realidad desde la encrucijada del mundo globalizado. Esto es así, porque la globalización no es una condición epistemológica de la teoría, sino una cualidad que afecta al ámbito que es objeto de estudio teórico (Touriñán, 2007)

\section{La Teoría de la Educación Agrupa Diversos Saberes Científicos}

La teoría de la educación se manifiesta en los últimos años con una temática muy abundante y agrupada en diversos saberes científicos como objeto propio de estudio (Medina Rubio, 1992, pp. 171-172). La teoría de la educación es una entidad con la que se representa una parte del conocimiento de la educación y pretende considerarse y presentarse a la sociedad como un logro epistémico muy importante. La teoría de la educación es parte del conocimiento de la educación, por tanto, según sea este conocimiento, la teoría de la educación será entendida de una u otra manera (Touriñán, 1987; Rodríguez, 2006).

Si examinamos un campo concreto de la teoría de la educación en una fase puntual de su desarrollo, nos encontramos con un cuerpo de información relativa al objeto de investigación. Ejemplos de campos estándar de investigación del conocimiento educativo sistematizado lo encontramos en Touriñán (1987, 1988 y 1989), Colom (1982, 1988), Escolano (1978), Sarramona (1991), Quintana Cabanas (1988 y 1995), García Carrasco y García del Dujo (1996), Ortega (2003), entre otros.

En este contexto se puede afirmar que la teoría de la educación posee un dominio para abordar los problemas con criterios y prioridades propias de las líneas de investigación que va consolidando como propias y compartidas en el contexto de la 
autonomía funcional, la dependencia disciplinar y la fecunda relación interdisciplinar (Touriñán, 1989; Touriñán y Sáez Alonso, 2006). Y el concepto de dominio tiene la riqueza de sentido que le da Suppe (1979) citado por Estany (1993, p. 182):

Un dominio no es meramente un cúmulo de información relacionada, sino que es un cúmulo de información relacionada acerca del cual hay un problema, generalmente bien definido y planteado sobre la base de consideraciones específicas. Además, dicho problema debe ser considerado importante (...) y digno de que se haga un esfuerzo para resolverlo. Además (...) debe ser susceptible de ser abordado en el nivel de desarrollo científico en que se esté.

Las teorías son, pues, una respuesta a los problemas surgidos en un dominio y el status epistemológico de las teorías les permite responder adecuadamente, es decir, según el nivel de desarrollo científico del momento, a cuestiones del dominio. "La Teoría de la Educación, como no podía ser de otro modo, se ocupa de conceptos problemáticos -el de educación- y de realidades controvertidas -el acto educativo-" (Puig Rovira, 1996, p. 167). De la misma manera, la teoría de la educación tiene un dominio de información sobre una problemática bien definida y planteada según el nivel de desarrollo científico susceptible de conocimiento. En esta tarea, la teoría de la educación busca constantemente una fundamentación cada vez más segura con el fin de garantizar un conocimiento pertinente de la educación.

La teoría de la educación agrupa, pues, diversos saberes científicos como objeto propio de estudio. Y su objetivo, como nivel de análisis con sentido epistemológico que es, consiste en elaborar enunciados teóricos sustantivos sobre la educación y proposiciones normativas específicas de intervención (Touriñán, 1987; Sáez Alonso, 1994, p. 232). O, en palabras de Larrosa (1990):"lo que interesa es qué hacen los pedagogos y los investigadores en educación para que sus productos tengan esa forma particular que les permita calificarlos como científicamente fundados" (p. 138).

Pero además, la teoría de la educación puede ser vista como una disciplina que está fundamentada epistemológicamente -por la forma de conocer- y ontológicamente -por el ámbito de realidad que estudia-. Y por eso sustenta a la educación y la hace susceptible de estudio con sentido autónomo. La autonomía funcional permite a la pedagogía "elaborar proposiciones teóricas sustantivas de la educación y proposiciones tecnológicas específicas de la educación” (Touriñán, 1987, p. 268) a través de un pautado y permanente interrogatorio del modo de encarar el sentido y meta de la intervención pedagógica (Davis, 1987).

En definitiva, la teoría de la educación investiga y analiza las investigaciones de y sobre la educación para que los educadores tengan unos productos que se les permita etiquetarlos como científicamente fundados. Es decir, cuando nos preguntamos qué es la teoría de la educación nos interrogamos sobre qué es lo que hacen los teóricos de la educación, cuáles son sus objetivos, contenidos y procedimientos y que se concretan en la práctica de la educación. A este respecto conviene recordar a Medina Rubio (1992) quien afirma que "la reflexión teórica de la educación ha de ser una teoría de la práctica y para la práctica. Una teoría que atiende a fundamentar la práctica de la que surge y a 
la que sirve y orienta” (p. 151). Y sin olvidar que toda práctica educativa debe estar sustentada en una teoría lógica, coherente y bien fundamentada, pues no se puede intervenir eficazmente sin un conocimiento teórico de la acción educativa en sus diferentes dimensiones que ayude a "la explicación, interpretación y transformación de la intervención pedagógica general” (Touriñán, 1987, p. 270).

Cada modo de entender el conocimiento de la educación ha generado un conjunto de conocimientos nada despreciables acerca de la educación. Las aportaciones de cada corriente son la base de su fuerza dentro del gremio de los profesionales de la educación. Parece evidente que el conocimiento de la educación reclama una respuesta amplia relativa a la relación método, metodología y modelo en cada problema a estudiar y resolver. Y así las cosas, es seguro que, según el tipo de problemas que estemos analizando, necesitaremos conocimiento de la educación distinto. A veces necesitaremos ciencia de la educación (para reglas y normas derivadas del proceso); a veces necesitaremos estudios científicos de la educación, teorías prácticas y teorías interpretativas (reglas para fines dados y orientaciones de la acción hacia determinados efectos que justifica la teoría interpretativa); y, por supuesto, necesitaremos estudios filosóficos de la educación, cuando queramos hacer fenomenología de un fin en sí, estudiar la lógica interna del fin dentro del sistema conceptual de "educación" o conocer las consecuencias que se derivan para la educación de una determinada concepción de vida (Touriñán, 1987; Touriñán y Rodríguez, 1993; Touriñán y Sáez Alonso, 2006).

Desde esta perspectiva, apertura y prescriptividad se convierten en objetivo de investigación compartido en el desarrollo de la disciplina de teoría de la educación y avala la profundización en la dimensión de la pluralidad de la investigación disciplinar en la cuádruple acepción de (Touriñán, 1989 y 2007a):

- Investigaciones teóricas acerca de la educación (teorías filosóficas e interpretativas de la educación).

- Investigaciones teóricas de la educación (teoría como nivel epistemológico de análisis).

- Investigaciones de teoría de la educación (teoría y tecnología de la intervención pedagógica general)

- Teorías prácticas de la educación (orientación de la acción combinando metas socialmente válidas y medios validados en las teorías interpretativas)

\section{La Reflexión Pedagógica se Produce en Todo Tipo de Situaciones las Refleja}

Es necesario recordar que la Educación como objeto de conocimiento no fue entendida siempre del mismo modo. Sin duda, las diferentes formas de comprender la educación producen distintos tipos de conocimiento de dicha realidad y, por consiguiente, estas distintas clases de entender la educación dan lugar a diferentes maneras de construir la teoría de la educación.

En la pedagogía y, más concretamente, en la teoría de la educación se observa un campo abierto sobre el conocimiento de la educación innovador, evolutivo, creciente y diversificado. La consecuencia de esto no es un relativismo. Como afirma Moulines: 
el relativismo (...) es una concepción que se despedaza automáticamente a sí misma, es suicidio intelectual. Muy al contrario de proporcionarnos un punto de vista más "progresivo", más "abierto" con respecto (...) al pensamiento, en general, el relativismo, en cualquiera de sus variantes, conduce a la parálisis espiritual, su corolario irremediable consiste en un silencio absoluto (Moulines, 1991, p. 27).

Este relativismo impondría a los teóricos de la educación dejar de pensar e investigar. Pues, lo que el relativista parece olvidar es que la mera constatación de la abundancia de ideas, métodos y definiciones en educación no da pie para concluir de manera alguna, afirmando la mera arbitrariedad, o proponiendo una inevitable equiparación entre todas las aportaciones en este campo de reflexión sobre el quehacer educativo.

Son muchas las definiciones de educación y los campos de investigación que se han producido a lo largo de estos últimos años. La lista puede ser interminable. Algunos de los objetos sobre los que ha recaído la consideración "educativamente" de un conocimiento innovador, evolutivo, creciente y diverso son: la persona, la educación como conocimiento, la motivación escolar, la escuela, la familia, la sociedad, la religión, la filosofía, las drogas, el trabajo, la economía, la política, la cultura, el profesor, la epistemología, los paradigmas de la investigación educativa, la reeducación de menores en desamparo/conflicto social, la teoría cibernética de la educación, el punto de vista tecnológico y artístico de la educación, la sociología de la educación, las políticas de la educación, la tecnología educativa, los valores y la educación, la globalización y la educación, la educomunicación, la formación a distancia con medios electrónicos (elearning, en inglés) que se basa en la utilización de Internet para facilitar la enseñanza y el aprendizaje, la metateoría pedagógica, etc. Y por supuesto la educación misma ( Sáez Alonso, 1998 y 2006).

El campo educativo y la teoría de la educación es ciertamente inmenso y una mirada sola no puede abarcarlo totalmente. Pero no por ello podemos aceptar al respecto la argumentación relativista. La argumentación relativista no nos sirve para la fundamentación de la teoría de la educación ni de ningún método con que desarrollarla debidamente. Además, todos los días surgen nuevas ideas y proposiciones: educación intercultural, educación para la ciudadanía, educación en las instituciones hospitalarias, educación virtual, educación en el ámbito residencial geriátrico, educación y ocio, educación sexual, educación deportiva, educación y tratamiento de conflictos, educación y bioética, educación emocional, educación pública, educación moral, etc.

Todos estos y otros más son ejemplos bien conocidos, extraídos de los libros y revistas: todos ellos son objeto de investigaciones educativas. Y, en realidad, esta lista no explicita todo aquello de lo que se han ocupado los pensadores desde la antigüedad: Platón, Aristóteles, Séneca, Quintiliano, Tomás de Aquino, Erasmo, Vives, Kant, Rousseau, Dewey... hasta los actuales pensadores. 


\section{Algunas Definiciones Sobre Educación que Generan Corrientes del Conocimiento de la Educación}

Puede resultar ilustrativo considerar esta diversidad de contenido, con el examen de significados distintos atribuidos por los autores a lo que supuestamente es el "mismo" objeto, cuando definen el concepto de educación.

Tomemos algunas definiciones (Sarramona, 1991, pp. 27-34; García Aretio, 1989, pp. 14-18): Platón: "Educar es dar al cuerpo y al alma toda la belleza y perfección de que son capaces". Alfonso X El Sabio definía la educación como una acción humana, la de "facer que los fijos lleguen al acabamiento de ser ommes", es decir, "Hacer que los hijos lleguen al acabamiento de ser hombres". Dilthey: "la educación es la actividad planeada por la cual los profesores forman la vida anímica de los seres en desarrollo”. Y sin ánimo de ser exhaustivos, observamos que la educación es perfeccionamiento, socialización, influencia y autorrealización, proceso activo de conducción y ayuda (Fermoso, 1994; Zubiri, 1992), desarrollo de las facultades humanas, comunicación (Sacristán, 1989); desarrollo perfectivo de la persona: "La educación es una modificación del hombre (...) esta modificación no tendría sentido si no fuera un perfeccionamiento, un camino hacia la perfección” (García Hoz, 1981, p.18). Y también, describe la educación como "perfeccionamiento intencional de las potencias específicamente humanas" (p. 27). "La educación es un "factum” que el hombre genera/realiza/como actividad/instrumento/ mecanismo para posibilitar/potenciar su configuración como especie e individuo de acuerdo con un "patrón" (Castillejo, 1994).

También, la educación es un proceso para alcanzar la madurez humana, es el intento de lograr un perfeccionamiento cada vez mayor, es la acción que genera un cambio a través de la influencia por la relación y la comunicación, orientada hacia el futuro; es proceso de aprendizaje que implica la inteligencia, la voluntad y la afectividad, orientado al uso y construcción de experiencia axiológica para decidir y realizar un proyecto personal de vida, dando respuesta de acuerdo con la oportunidades a las exigencias que se plantean en cada situación; es un proceso, en definitiva, con sentido integral, personal y patrimonial (Touriñán, 1984 y 2006). Para Durkheim (1976) la educación "es la acción ejercida por las generaciones adultas sobre las que no están todavía maduras para la vida social" (p. 98). "La educación es el influjo elevador, integrado por cuidados psíquicos (liberación de trabas, enseñanza, inspiración, ejercicios) que la generación adulta ejerce sobre el desarrollo de la que está madurando, con objeto de preparar a los individuos que la integran a conducir personalmente su existencia dentro de las sociedades que le circundan vitalmente y con ello a la inteligente realización de los valores en que se fundan dichas sociedades" (Göttler, 1955, p. 72).

La educación es una interferencia consciente de uno o varios agentes en el desarrollo de los hechos educativos (Escámez, 1988). La educación es una producción, un rendimiento, una actividad interna a los propios sujetos de la relación educativa 
(Medina Rubio, 1992, p. 128). Para Lemus (1973) "el fin educativo es la formación de hombres libres, conscientes y responsables de sí mismos, capaces de su propia determinación" (p. 15).

La educación es una acción educativa realizada por una intervención racional y técnica sobre el educando y que genera aprendizaje (García Carrasco y García del Dujo, 1966). Para Colom y Núñez Cubero (2001) "educar es desarrollar un proceso permanente -mediante aprendizaje- de formación personal, social y cultural orientado por un sistema axiológico y moral que propicie la capacidad crítica y de adaptación innovadora en el hombre” (p. 22). La educación es un proceso racional (Escámez, 1988) que responde al qué y al para qué del proceso educativos. La educación debe emplear, a la hora de la intervención, el criterio de racionalidad científico-técnica. La educación es una acción deliberativa e intencional dirigida a optimizar a la persona propia del profesional de la educación que "es un especialista competente para realizar elecciones técnicas y, por tanto, sabe qué cambios son pedagógicos y cuáles pueden lograrse en un determinado sujeto y deben (cursiva del original) lograrse con fundamento de elección técnica" (Touriñán, 1987, p. 160).

Y es que en educación los contenidos especulativos, finalísticos, normativos, técnicos y artísticos (Medina Rubio, 1992, pp. 147-149), la estructura semántica, los fines, la concepción, el proceso, el llevarlos a cabo, su resultado y su producto son tan diversos, que resulta necesario fundamentarlos. Unos ven la educación como superación ética de los instintos, como proceso espiritual, como desarrollo de las cualidades innatas, como arte y desarrollo de los fines de vida deseables o como justificación moral de conductas singulares (Morando, 1969), otros la describen como influencia social del profesorado y hay quienes entienden la educación como sistema de comunicación concebido cibernéticamente y virtualmente. En suma, son distintas formas de ver la educación que generan conocimiento de la educación procedente de muy diversas formas de conocimiento. Y todos ellos son necesarios.

\section{Ningún Tema de Educación le Resulta Extraño a laTeoría de la Educación}

Si nos detenemos en este repaso somero de algunas definiciones podemos observar que la educación, en un momento, es analizada como parte de la sociedad. Así la educación es coherente con la estructura social que se da en dichas sociedades. Para Rodríguez, que sigue las tesis iniciadas en 1987 por el profesor Touriñán este tipo de educación está relacionado con los grandes tratados de la concepción general de la Educación donde se definen la sociedad ideal y el tipo de educación que se debe desarrollar para alcanzarla, es decir, "en qué valores se deben formar los miembros de esa sociedad para alcanzar la sociedad ideal. Estamos, por tanto, hablando de un conocimiento de tipo filosófico cosmovisionario" (Rodríguez, 2006, p. 33).

En este marco de interpretación de cómo es el conocimiento de la educación, el conocimiento de la educación se centra en el estudio de las consecuencias que se derivarían de cada cosmovisión o de la concepción general de la educación que se postula "a priori". O dicho de otro modo "se centraría en el estudio normativo de los 
fines de vida en los que deben formarse los hombres (sic) " (Rodríguez, 2006, p. 37). Es un conocimiento filosófico de la educación. La educación debe resolver en su inicio fundamentalmente el problema del fin, permaneciendo en un conocimiento teórico sobre la justificación del fin de la educación y el estudio normativo de los fines. Por consiguiente, la teoría proporciona las finalidades de vida deseables e identifica la finalidad de vida deseable, conociendo las consecuencias que se derivan para la educación de una determinada concepción de vida.

En esta corriente, los estudios se centran en los fines de vida deseables para los hombres y mujeres y no, en las metas pedagógicas ni en las reglas de intervención. De esta forma, la educación se entiende como justificación moral de conductas singulares. Como afirma Touriñán (1989) en este marco de interpretación de cómo es el conocimiento de la educación no existen pautas intersubjetivables que nos permitan analizar las diferentes reglas de intervención, porque la teoría no explica lo que se tiene que hacer en una buena práctica.

Pues bien, la teoría de la educación, en este estudio normativo de los fines de vida deseables, está claro que se identificará con las filosofías o teorías filosóficas de la educación (Touriñán, 1989; Fullat, 1979; Rodríguez, 2006).

Frente a este tipo de conocimiento surge otra forma de entender la educación como objeto de conocimiento. La preocupación de esta corriente es entender la educación como objeto de estudio específico. Se pretende, en este caso, construir un conocimiento de la educación de tipo científico frente al conocimiento filosófico de la postura anterior.

Este posicionamiento parte del convencimiento de que los fenómenos y acontecimientos educativos son diferentes a los acontecimientos y fenómenos de otros ámbitos de la realidad, en otras palabras, la educación es entendida como un ámbito de realidad susceptible de ser conocido y, como apuntamos, de conocimiento científico (Rodríguez, 2006, p. 34).

Este posicionamiento da lugar a dos tendencias. La primera de ellas entiende que los fenómenos educativos son complejos, se pueden descomponer en fenómenos más simples que se producen en otros ámbitos de la realidad y, por tanto, de otros objetos de conocimiento y desde los cuales se reflexiona sobre la educación, por ejemplo, desde la psicología de la educación, la sociología de la educación, la antropología de la educación, la economía de la educación. De ahí que el conocimiento de la educación se pueda construir desde esta perspectiva, recopilando lo que los diferentes conocimientos elaboraron sobre los fenómenos simples que dan lugar a los acontecimientos y fenómenos educativos (Touriñán y Rodríguez, 1993).

Esta corriente identifica la teoría de la educación con la mentalidad subalternada como la llama Rodríguez (2006). Aquí, la educación es un objeto de estudio genuino. Ahora bien, "la educación es simplemente un marco de referencia que se resuelve utilizando las disciplinas generadoras" (p. 40). El conocimiento de la educación se resuelve desde cada una de sus dimensiones particulares apoyándose en disciplinas que poseen una estructura teórico-conceptual consolidada. El conocimiento de la educación 
que se obtiene es subalternado porque la validez de las reglas construidas para intervenir viene dada por la validez de las vinculaciones establecidas en las disciplinas generadoras y en la utilización de los conocimientos de las disciplinas consolidadas como puede ser la psicología, la economía, la sociología, la antropología, la biología ... de la educación. Los conocimientos teóricos y prácticos validados en las disciplinas generadoras quedan validados para la educación.

En este sentido, la función pedagógica es una tarea susceptible de conocimiento científico; queda validada por medio de las vinculaciones que las diferentes teorías interpretativas de las disciplinas generadoras establecen y la intervención ya no se resuelve con la intuición y la experiencia, sino que se resuelve utilizando los principios de las disciplinas generadoras. Conviene recordar finalmente que los conocimientos elaborados al amparo de las disciplinas científicas anteriormente citadas son necesarios según el tipo de problemas que se estén planteando e intentando resolver en la educación.

La segunda tendencia también entiende que los fenómenos y acontecimientos educativos son complejos y pueden descomponerse en fenómenos más simples. Pero a diferencia de la tendencia anterior, no considera que la educación pueda estudiarse, analizarse y comprenderse como objeto de conocimiento que es, desde las explicaciones que otros acontecimientos hacen de esos fenómenos y acontecimientos simples (economía de la educación, sociología de la educación, etc. ) sino que debe estudiarse para "construir un conocimiento propio, donde la explicación de los fenómenos educativos tenga significación intrínseca a la Educación” (Rodriguez, 2006, p. 34).

\section{LaTeoría de la Educación Tiene Significación Intrínseca en sus Términos}

En este trabajo, se apuesta por una teoría de la educación que trate de elaborar un conocimiento donde los términos educativos tengan significación intrínseca al ámbito de realidad educación. Desde esta óptica, la educación no quiere ser un marco de referencia a resolver en los términos de las disciplinas generadoras, descritas anteriormente, sino que se quiere afirmar un ámbito de realidad con significación intrínseca en sus términos.

Por consiguiente, la teoría de la educación se comprende como un ámbito científico autónomo "porque se construye en función de su propio objeto de estudio (la educación) utilizando la forma de conocimiento científico-tecnológico, al igual que las demás disciplinas científico-autónomas más consolidadas como pueden ser la física, la biología, la psicología, la sociología” (Rodríguez, 20006, p. 45). Por ello, la teoría de la educación utiliza la racionalidad científico-tecnológica en el ámbito de la educación, entendida como realidad con significación intrínseca en sus términos (Touriñán, 1989; Bunge, 1981). Su meta es elaborar teorías sustantivas y tecnologías específicas de la educación, es decir, tener en cuenta su validez teórica según la cual en términos epistemológicos y metodológicos una forma de conocimiento es válida y también tener en cuenta su validez práctica de los objetivos y propósitos de la educación. 
Para Touriñán (1989) esta autonomía es funcional y compatible con la defensa del principio de dependencia disciplinar y es propiciadora de una fecunda relación interdisciplinar. La teoría de la educación, al igual que las demás disciplinas sustantivas, utiliza y desarrolla la forma de conocimiento científico-tecnológico, que epistemológicamente tiene tres niveles de análisis: el teórico, el tecnológico y el práctico. Es decir,

se compone de teorías sustantivas de la educación que establecen vinculaciones entre condiciones y efectos que afectan a un acontecimiento educativo, por medio de términos, enunciados y conceptos con significación intrínseca al ámbito educativo. De tecnologías específicas de la educación, que construyen reglas de intervención para alcanzar objetivos intrínsecos o metas educativas a partir de las vinculaciones establecidas en las teorías sustantivas de la educación. Y de intervención pedagógica, como el ajuste de la regla de intervención al caso concreto, o lo que es lo mismo, la puesta en práctica de lo que se establece en las teorías sustantivas y en la tecnología específica (Rodríguez, 2006, p. 46).

En este sentido y para este autor, la teoría tiene como función explicar el modo de intervenir, al establecer las vinculaciones que existen entre las condiciones y los efectos que se asocian a una intervención. Y, al mismo tiempo, la práctica, en el contexto de justificación de la acción, rige a la teoría, porque son los fenómenos que acontecen en cada intervención los que sirven de elemento de contraste para comprobar si la teoría da cuenta ajustada de los acontecimientos ocurridos (Touriñán, 1989).

En esta corriente o marco de interpretación de cómo es el conocimiento de la educación, la función pedagógica se entiende como la puesta en acción de las intervenciones realizadas por la teoría de la educación. Por consiguiente, la función pedagógica es especializada y específica, y a diferencia de la corriente en la que utilizaba los principios de las disciplinas generadoras, y ella era subalterna, aquí es generadora de principios y teorías sustantivas y tecnologías específicas del ámbito o sector de la educación que estudian.

Se puede entender, por lo tanto, que a la teoría de la educación le corresponde desarrollar el conocimiento científico-tecnológico del área de la educación que se corresponda con la intervención pedagógica general y con las dimensiones generales de intervención. Es decir, la teoría de la educación "se ocupa de los problemas de explicación, interpretación y transformación de la intervención pedagógica general, o lo que es lo mismo, que se ocupa de la teoría y de la tecnología de la intervención pedagógica general” (Touriñán, 1989, p. 22).

Todos esos conocimientos (filosófico, subalternado o científico-tecnológico) son conocimientos de Educación, porque todos tratan de explicar, analizar y comprender los fenómenos y acontecimientos educativos. Por ello, ningún tema de educación le resulta extraño a la teoría de la educación. Y, por consiguiente, el conocimiento de la educación crece en función de cómo se entienda la educación como objeto de conocimiento, es decir, según que las respuestas se adapten a la tendencia de 
la consideración de la educación como objeto de estudio, al tipo de conocimiento a obtener para saber educación, al modo de resolver el acto de intervención educativa y a la posibilidad o no de estudio científico y de la ciencia de la educación" (Rodríguez, 2006, pp. 35-36).

Lo dicho hasta aquí parece constituir, con lagunas y carencias, por supuesto, un "gran bazar del mercado pedagógico" (Sáez Alonso, 1994, p. 235). Y es una constatación acerca de los contenidos, argumentos y definiciones que configuran la educación; una constatación, no de lo que la educación debe ser, sino de lo que es y de las situaciones en las que se refleja. Esta comprensión, que es necesario que sea atenta y crítica, influye en el camino que tome la teoría de la educación de forma responsable y abierta para responder adecuadamente de forma no ilusoria, pero siempre limitada y con riesgo de error.

\section{Búsqueda sin Término en la Construcción del Conocimiento de la Educación}

Lo que compete a los investigadores de la teoría de la educación es intentar orientarse bien, "pues hay caminos y no todos los caminos son iguales. La diferencia estriba sobre todo en determinar a dónde queremos ir. Esta es la cuestión radical de principio" (Moulines, 1991, p. 30). Es necesario determinar a dónde se quiere llegar con la educación, qué es lo que se pretende con esta actividad que hemos denominado educar y exponerla de manera clara y honesta. Se observa que es un asunto demasiado complejo, y que nadie, solo, ni tampoco muchos y en equipo han captado su totalidad. Imposible es presentar semejante riqueza de paisaje en una sola fotografía. La razón es que la educación está en continuo proceso de replanteamiento por su complejidad y por la importancia decisiva de sus cuestiones. $\mathrm{Ni}$ toda respuesta vale, ni las mejores llegan a ser definitivas. Aquí actúa la capacidad recursiva del pensamiento humano a que nos hemos referido anteriormente.

La teoría de la educación se enfrenta a una problemática tan compleja y abarcadora como la de dar cuenta de las diversas parcelas de la realidad con sentido y operatividad educativos y

debe ser capaz no sólo de explicar científicamente los procesos (educativos) sino también poseer la suficiente potencia científica para elicitar legalidades que los regulen y para reintegrar los nuevos datos y enunciados básicos, permitiendo en consecuencia progresivas reformulaciones más específicas y adecuadas (Castillejo, 1985, p. 51).

Es decir, la teoría de la educación trata de captar lo esencial de la ciencia pedagógica, "habla, discute, valora o evalúa aquello que los científicos o la comunidad científica -de pedagogos- (añadido nuestro) están diciendo o haciendo" (Oldroyd, 1993, p. 9). La teoría de la educación pone de manifiesto la importancia del tema de "la educación como objeto de conocimiento" (Tourinán y Sáez Alonso, 2006, p. 378) fortaleciendo la 
relación entre la función y la intervención pedagógicas y pretendiendo que el conocimiento de la educación sea adecuado para explicar, interpretar y decidir la intervención pedagógica, estableciendo parámetros acerca del conocimiento de la educación y de los diferentes posiciones que se mantienen respecto del conocimiento de la educación.

Así las cosas, sigue siendo importante insistir en que la teoría de la educación debe estar al lado de las aportaciones en aquellos niveles en que la ciencia se pone más a la altura de sus posibilidades, deteniéndose más en los movimientos epistemológicos actuales que se han ocupado y que han influido en la metodología científica moderna. El rigor metodológico es garantía del conocimiento en cada ciencia y por ello los métodos de investigación constituyen un elemento esencial en el desarrollo y creación del conocimiento de la educación. En palabras de Arturo de la Orden (1985)

los métodos propios de adquisición de conocimientos constituyen un componente esencial de todo ciencia (...) y (...) los grandes avances científicos han ido unidos a cambios importantes (avances) en la metodología; precisamente por eso puede decirse que existe correlación positiva entre ciencia y método, a la hora de construir el conocimiento de la educación (p. IX).

En este trabajo, los objetivos son analizar los ámbitos que nos ayuden a comprender el papel de la teoría de la educación en la construcción del conocimiento de la educación. $\mathrm{Y}$ es establecer los ámbitos que nos permiten, con criterio lógico, comprender la distinta consideración que el conocimiento de la educación tiene.

Se intenta describir los marcos científicos, sociales y educativos que hagan que la teoría de la educación sea un instrumento de creación de conocimiento de la educación y que justifique que ésa es la investigación que debe hacerse del objeto de conocimiento "educación" (Touriñán y Sáez Alonso, 2006). De forma racional porque el conocimiento educativo es fruto de una actividad interpretativa de la razón fundada en la experiencia y de forma crítica "porque las interpretaciones de la razón, aunque encaminadas a conocer la realidad objetiva, no la expresan exhaustiva y perfectamente, debiendo ser siempre corregidas y revisadas críticamente" (Monserrat, 1984, p. 80).

Sin duda alguna, la labor de la teoría de la educación consiste en tratar de elaborar descripciones teóricas, terminológicas y prácticas, así como términos, enunciados y conceptos de la realidad educativa cada vez más ajustados a la misma, sabiendo que no podemos observar la realidad, los fenómenos educativos, sin juicios previos, es decir, sin un marco conceptual en el cual dar sentido a nuestras observaciones. Y, sabiendo que, toda percepción y observación educativa, supone siempre una interpretación y una teoría.

Parafraseando a Heisenberg, podemos invocar aquella máxima suya, según la cual "no conocemos la realidad, sino la realidad sometida a nuestro modo de interrogarla". Y sobre nuestras experiencias observacionales "directas" o "inmediatas", afirma Popper (1974): "Hay que admitir que, en general, estas experiencias son perfectamente "buenas" y "eficaces", pero no son ni directas ni inmediatas, ni mucho menos fiables" ( p. 75). Y en otro lugar afirma Popper (1977) que "las observaciones son siempre 
interpretaciones de hechos observados, no hechos puros; es decir, son interpretaciones a la luz de teorías" (p. 103).

Se trata, en resumen, de indicar las condiciones en que se manifiesta el conocimiento de la educación, investigando científicamente, de forma objetiva, aunque parcial y provisional, pero la única posible en un momento específico, aquella que presenta una mayor adecuación a la realidad. La ciencia educativa, la teoría de la educación y su propio objeto de estudio, la Educación, no es un saber absolutamente seguro, ni pretenderá la ilusoria meta de que sus respuestas sean definitivas, sino siempre provisionales y con contrastaciones constantemente renovadas y cada vez más rigurosas, pues como afirma Popper (1977)

La ciencia nunca persigue la ilusoria meta de que sus respuestas sean definitivas, ni siquiera probables; antes bien, su avance se encamina hacia una finalidad infinita y, sin embargo, alcanzable: la de descubrir incesantemente problemas nuevos, más profundos y más generales y de sujetar nuestras respuestas (siempre provisionales) a contrastaciones constantemente renovadas y cada vez más rigurosas (p. 262).

La teoría de la educación adoptará, además, una actitud de humildad científica ante la imposibilidad lógica y epistemológica de asentar nuestros conocimientos en la seguridad absoluta. Por eso, el crecimiento del conocimiento de la educación que se construye y que aporta la teoría de la educación se basará en la permanente crítica de la razón, en el constante sometimiento a prueba de todas las teorías sobre la educación y en la confrontación permanente de estas con los hechos predichos por las mismas. Como dice el título de la autobiografía de Popper: Búsqueda sin término, la ciencia no es posesión de la verdad, sino búsqueda incesante, crítica, sin concesiones, de la misma (Mardones, 1991, p. 37).

A través de estos principios y si vale para algo, la teoría de la educación servirá para la comprensión, explicación y transformación de la educación en un mundo globalizado. Señalará los métodos potenciadores de conocimiento educativo y, al mismo tiempo, opondrá la consideración del método de investigación como un "molde" fijo y previo al que debe ajustarse cualquier investigador en educación, abandonando, incluso, los métodos existentes si son un obstáculo para el avance científico de la educación, e inventará otros, estableciendo principios genéricos en la construcción de la ciencia de la educación y mostrando "la complejidad, la inconmensurabilidad y la inagotabilidad de la realidad” (Bueno, 1973, p. 305) y, por supuesto, de la realidad de la educación.

El cambio de contexto en las formas de entender el ámbito de realidad educación creado por el flujo de ideas nuevas, por la globalización, es como el horizonte, visto desde la cubierta de un barco que navega: siempre cambia, pero aparentemente sigue siendo el mismo, hasta que se presenta a la vista una nueva isla o costa. Pasado el horizonte, al mirar atrás, están las viejas teorías educativas descartadas; delante, están las nuevas tierras. El horizonte que tenemos delante define el conjunto actual de expectativas teóricas y los problemas que podemos ver ahora. Pero estos, a su vez serán descartados y olvidados (Young, 1993, p. 34). 
La teoría de la educación ofrecerá, por tanto, un criterio científico sobre cuestiones vitales de desarrollo del conocimiento científico-tecnológico y de la intervención pedagógica general en un mundo global, sabiendo que la ciencia se ha convertido en el eje de la cultura contemporánea. Y, por ser el motor de la tecnología, la ciencia ha venido a controlar indirectamente la economía de los países. Solamente una teoría de la educación basada en la ciencia, fomentará el desarrollo integral e ininterrumpido de las personas en la sociedad actual.

Los problemas en la educación son el muelle que impulsa la actividad científica investigadora. Una vez planteado y examinado un problema o un sistema problemático, se busca una solución. Pero advierte Bunge (1981):

No hay un problema científico que se resuelva precipitándose sin más hacia el laboratorio (...) Las experiencias científicas no tienen lugar en el vacío: se proyectan con ideas determinadas y se interpretan con ayuda de teorías (...) Vale la pena, antes de estudiar la experiencia científica, examinar las ideas científicas, conjeturas llamadas hipótesis, hipótesis ascendidas a leyes y sistemas de leyes llamadas teorías (p. 248).

En este punto del trabajo tiene sentido decir que hablar de "conocimiento de la educación" es lo mismo que interrogarse acerca de la educación como objeto de conocimiento, lo que equivale a interrogarse sobre qué es lo que hay que conocer para entender y dominar el ámbito de la educación; o lo que es lo mismo, cuáles son los componentes del fenómeno educativo que hay que dominar para entender dicho fenómeno (Touriñán, 1987). La teoría de la educación desempeña un papel singular en este cometido. Para definir la educación como objeto de conocimiento es necesario analizar los diferentes modelos de evolución del conocimiento de la educación. Estos análisis dan lugar a esquemas de interpretación que permiten comprender la diferente consideración que el conocimiento de la educación tiene o ha tenido. Con otras palabras, se trata de descubrir las propiedades que nos permitan caracterizar los diferentes momentos de la consideración de la educación como objeto de conocimiento. Es decir, saber cuáles son las propiedades que definen a la educación como objeto de conocimiento en los diferentes momentos y, además cómo se justifica que esa es la investigación que tiene que realizarse del objeto de conocimiento educación. Este tipo de planteamientos se establecen ordinariamente bajo la denominación genérica de "paradigma de investigación” (Khun, 1975 y 1978; Bachelard, 1973; Touriñán, 1987; Rodríguez, 2006; Sáez, 2005; Touriñán y Sáez Alonso, 2006).

\section{A Modo de Conclusión}

El conocimiento de la educación procede de muy diversas fuentes de conocimiento y genera, a su vez, diferentes formas del mismo. Una fuente primaria que genera conocimiento educativo es la teoría de la educación y que es parte del conocimiento de la Educación. 
La teoría de la educación tiene un papel específico en la investigación educativa y posee un dominio para abordar los problemas de educación con criterios propios y está fundamentada epistemológicamente, por la forma de conocer y ontológicamente, por el ámbito de la realidad que estudia. Por eso sustenta a la educación y la hace susceptible de estudio con sentido de autonomía funcional, utilizando y desarrollando el ámbito de realidad que es la educación como objeto y como meta de su quehacer.

Ningún tema de educación le resulta extraño a la teoría de la educación y en el mundo global en que vivimos, la teoría de la educación se enfrenta a una problemática compleja que exige poseer la suficiente potencia científica para establecer parámetros acerca del conocimiento de la educación. No se trata de vivir de los resúmenes de los libros y de las investigaciones que otras ciencias han realizado, sino de que la teoría de la educación debe estar al lado de las aportaciones en aquellos niveles en que la ciencia se pone más a la altura de sus posibilidades, deteniéndose más en los movimientos epistemológicos actuales que se han ocupado y que han influido en la metodología científica moderna. Este tipo de planteamientos se establecen ordinariamente bajo la denominación genérica de paradigmas de investigación.

En este contexto se puede afirmar que uno de los conceptos introducidos en el ámbito de la teoría de la ciencia para clarificar y clasificar lo que significa y es científico, resulta ser el de paradigma. A su vera, la teoría de la educación tiene el reto de permanecer abierta a la investigación para favorecer su desarrollo como construcción científica y como disciplina académica bien sea como investigación acerca de la educación como ámbito de realidad, bien sea como investigación del conocimiento de la educación.

\section{Bibliografía}

Bachelard, G. (1973). Epistemología. Barcelona: Anagrama.

Bueno, G. (1973). Estatuto gnoseológico de las Ciencias Humanas. Fundación Juan March. Programa: Filosofía. Inédito.

Bunge, M. (1981). Investigación científica. Su estrategia y su filosofia. Barcelona: Ariel.

Castillejo, J. L. (1985). Sugerencias para una teoría de las ciencias pedagógicas. Papers d'educació (II), 46-56.

Castillejo, J. L. (1994). La acción educativa. En J.L. Castillejo y otros, Teoría de la Educación (pp. 29-44). Madrid:Taurus.

Colom, A. J. (1982). Teoría y metateoría de la educación. Un enfoque a la luz de la teoría general de sistemas. México: Trillas.

Colom, A. J. (1988). La calidad de la educación desde la teoría pedagógica y la historia. Bordón, 40, 2, 163-175.

Colom, A. J. y Nuñez Cubero, L. (2001). Teoría de la Educación. Madrid: Síntesis.

Davis, W.K. (1987). Educational research in the professions: Paradigms, peer review and promise. Professions Education Research Notes, 9 (1), 4-9.

Durkheim, E. (1976). Educación como socialización. Salamanca: Sígueme.

Escamez, J. (1988). La teoría pedagógica y el proceso educativo. En IX Congreso Nacional de Pedagogía, La calidad de los centros educativos (pp. 11-25). Alicante: Sociedad Española de Pedagogía

Escolano, A. y otros (1978). Epistemología y Educación. Salamanca: Sígueme.

Estany, A. (1993). Introducción a la filosofía de la ciencia. Barcelona: Crítica. 
Fermoso, P. (1994). Pedagogía Social. Fundamentación cientifica. Barcelona:Herder.

Fullat, O. (1979). Filosofías de la educación. Barcelona: CEAC.

García Aretio, L. (1989). La Educación. Teorías y conceptos. Perspectiva integradora. Madrid: Paraninfo.

García Carrasco, J. y García del Dujo, A. (1996). Teoría de la Educación. Educación y acción pedagógica. Salamanca: Universidad de Salamanca.

García Hoz, V. (1964). Diccionario de Pedagogía. Barcelona: Labor.

García Hoz, V. (1981). Principios de pedagogía sistemática. Madrid: Rialp.

Gimeno Sacristán, J. (2001). El significado y la función de la educación en la sociedad y cultura globalizadas. Revista de Educación, número extraordinario, 121-142.

Göttler, J. (1955).Pedagogía sistemática. Barcelona: Herder.

Khun, T.S. (1975). La estructura de la revoluciones cientificas. México: Fondo de Cultura Económica.

Khun, T. S. (1978). Segundos pensamientos sobre paradigmas. Madrid: Tecnós.

Larrosa, J. (1990). El trabajo epistemológico en Pedagogía. Una propuesta constructiva. Barcelona: PPU.

Lemus, L.A. (1973). Pedagogía. Buenos Aires: Losada.

Mardones, J. M. (1991) Filosofía de las ciencias humanas y sociales. Materiales para una fundamentación científica. Barcelona: Anthropos.

Medina Rubio, R. (1992). Explicación, norma y praxis en el conocimiento de la Educación (T.1). En R. Medina Rubio, T. Rodriguez Neira y L. García Aretio, Teoría de la Educación 1 (pp. 157-175). Madrid: UNED.

Monserrat, J. (1984). Epistemología evolutiva y teoría de la ciencia. Madrid: U.P.C.M.

Morando, D. (1969). Pedagogía. Historia crítica de las corrientes pedagógicas que han forjado el desarrollo de la educación. Barcelona: Luis Miracle.

Moulines, C. V. (1991). Pluralidad y recursión. Estudios epistemológicos. Madrid:Alianza.

Oldroyd, D. (1993). El arco del conocimiento. Introducción a la historia de la filosofía y metodología de la ciencia. Barcelona: Crítica.

Orden, A. de la (1985). Introducción. En A. de la Orden (Coord.), Diccionario de ciencias de la educación. Investigación educativa ( $p p$. IX-XXXVI). Madrid: Anaya.

Ortega, P. (2003). Teoría de la Educación, ayer y hoy. Murcia: Universidad de Murcia.

Popper, K. R. (1974). Conocimiento objetivo. Madrid: Tecnos.

Popper, K. R. (1977). La lógica de la investigación científica. Madrid: Tecnos.

Puig Rovira, J. M. (1996). La teoría de la educación y su concepción antinómica. En Varios, Por una Pedagogía Humanista. Homenaje al Profesor José María Quintana Cabanas ( pp.163-177). Madrid: Dykinson/Narcea.

Quintana Cabanas, J. Ma (1988). Teoría de la Educación. Concepción antinómica de la educación. Madrid: Dykinson.

Quintana Cabanas, J. Ma (1995). Teoría de la Educación. Concepción antinómica de la educación. (Nueva edición reformada). Madrid: Dykinson.

Rodriguez, A. (2006). Conocimiento de la educación como marco de interpretación de la Teoría de la Educación como disciplina. Tendencias pedagógicas, 11, 31-53.

Sacristán, D. (1989). Comunicación. En F. Altarejos y otros, Filosofia de la educación hoy (pp. 35-50). Madrid: Dykinson.

Sáez Alonso, R. (1994). Interrogación sobre el sentido y las bases de una metateoría pedagógica. Revista Complutense de Educación, 5(2), 231-248.

Sáez Alonso, R. (1998). La ciberpedagogía y la investigación educativa ante el nuevo milenio. Revista Complutense de Educación, 9 (1), 85-99.

Sáez Alonso, R. (2005). Bases metodológicas de la investigación científica y paradigmas. Revista Complutense de la Educación, 16 (I), 307-337.

Sáez Alonso, R. (2006). La educación intercultural. Revista de Educación ( M.E.C.), 339, 859-881. 
Sarramona, J. (1991). Fundamentos de educación. Barcelona: Ceac.

Touriñán, J. M. (1984). Delimitación de la acción educativa como acción. Revista Española de Pedagogía, 163, 79-97.

Touriñán, J. M. (1987). Teoría de la educación. Madrid: Anaya.

Touriñán, J. M. (1988). El conocimiento pedagógico: corrientes y parámetros. Educar, (14-15), 81-92.

Touriñán, J. M. (1989). Teoría de la Educación: identificación de la asignatura y competencia disciplinar. Revista de Ciencias de la Educación, 35 (137), 7-35.

Touriñán, J. M. (2006). Educación en valores y experiencia axiológica: el sentido patrimonial de la educación. Revista Española de Pedagogía (64: 234) 227-248.

Touriñán, J. M. (2007). Cooperación universitaria interregional: sinergias académicas desde la teoría de la educación para su desarrollo como disciplina a enseñar, a investigar y de investigación. Primer Seminario Luso-español de profesores de Teoría de la Educación. Guarda, Portugal. Discurso policopiado.

Touriñán, J. M. (2007a). Teoría de la educación: reflexiones en torno a la investigación disciplinar para el encuentro de guarda. Primer Seminario Luso-español de profesores de Teoría de la Educación. Guarda, Portugal. Discurso policopiado.

Touriñán, J. M. y Rodríguez, A. (1993) Significación del conocimiento de la educación. Revista de Educación 302, 165-192.

Touriñán, J. M. y Sáez Alonso, R. (2006). La metodología de investigación y la construcción del conocimiento de la educación. Revista Galega do Ensino, 48, 377-410.

Young, R. (1993). Teoría crítica de la educación y discurso en el aula. Barcelona: Paidós.

Zubiri, X. (1992). Sobre el sentimiento y la volición. Madrid: Alianza. 